Research Journal of Applied Sciences, Engineering and Technology 5(24): 5566-5571, 2013

DOI:10.19026/rjaset.5.4237

ISSN: 2040-7459; e-ISSN: 2040-7467

(C) 2013 Maxwell Scientific Publication Corp.

$\begin{array}{lll}\text { Submitted: October 12, } 2012 & \text { Accepted: December 03, } 2012 & \text { Published: May 30, } 2013\end{array}$

Research Article

\title{
Detection of Brain Activity in Functional Magnetic Resonance Imaging Data using Matrix Factorization
}

\author{
${ }^{1}$ Amir A. Khaliq, ${ }^{2}$ I.M. Qureshi, ${ }^{1}$ Ihsanulhaq and ${ }^{1}$ Jawad A. Shah \\ ${ }^{1}$ Department of Electronic Engineering, International Islamic University Islamabad \\ ${ }^{2}$ Department of Electronic Engineering Air University, ISSS Islamabad, Pakistan
}

\begin{abstract}
Non-negative matrix factorization (NMF) is becoming a popular tool for decomposition of data in the field of signal and image processing like Independent Component Analysis (ICA). In this study we are relaxing the requirement of non-negative data for NMF making the update equations simple and thus making it Matrix Factorization (MF) and implementing it on simulated Functional Magnetic Resonance Imaging (fMRI) data for detection of neuronal activity. Simulated fMRI data is processed to detect the hidden sources of task related activity, functional activity and artifacts using the proposed MF technique. Performance of the proposed scheme is better than NMF in terms of average correlation results of the extracted sources/time courses with the actual sources/time courses. Similarly proposed MF is computationally cost effective and converges fast as compared to NMF. Also extracted sources obey no permutation which is the limitation of ICA and NMF.
\end{abstract}

Keywords: Blind source separation, Functional Magnetic Resonance Imaging (fMRI), matrix factorization, medical image processin

\section{INTRODUCTION}

Functionality of brain can be detected by many techniques like Positron Emission Tomography (PET), Single Photon Emission Computed Tomography (SPECT) and fMRI etc. fMRI has the advantage of being non-invasive. In studying functionality of brain, subjects are asked to perform some visual or motor task and changes in BloodOxygen-level-Dependent (BOLD) signal are recorded in the form of 3-D images against time using fMRI scanner (Ogawa et al., 1990). These images formed by small variations of BOLD signal exhibits low SNR (Friman et al., 2001). This multi-image data is then analyzed for detecting the activated neurons of the brain responsible for the experimental task being performed during the test. Before starting the analysis, some preprocessing steps like de-noising (Khaliq et al., 2012), zero mean and whitening etc are also performed as a pre-requirement of statistical method being used for analysis. Statistical analysis techniques normally used for fMRI data are broadly divided into two main categories, that is, model driven approaches and data driven approaches. In model driven approaches, we need the experimental model of the fMRI test as in the case of time frequency analysis (Mitra et al., 1997), Statistical Parametric Mapping (SPM) (Friston, 1996), Canonical Correlation Analysis (CCA) (Friman et al., 2001) etc. Data driven approaches do not require any a priori knowledge of the BOLD experiment as in the case of Principal Component Analysis (Backfrieder, 1996), Independent Component Analysis (Hyvärinen et al., 2001), NMF (Lee and Seung, 1999) etc. However, a little knowledge of the data is required, which is being processed so that it can be decided that it obeys the validity of the data driven approach.

ICA is one of the promising method used for Blind Source Separation (BSS) problem in many fields, like communications and image processing (Conceição et al., 2011; Yu and Cheng, 2012), audio processing (Torkkola, 1999), fMRI (Vince et al., 2009; Wei et al., 2011) etc. However, one main limitation of ICA is the requirement of independence of sources. Some time this requirement cannot be fulfilled by the data, which is being processed for BSS problem. If it is the case in fMRI data, then one needs to look for other methods which do not require the independence of sources, like PCA and NMF etc. In PCA the assumption being made is that the sources of interest in the data are uncorrelated both in temporal and spatial domain (Xiaoxiang et al., 2004). NMF further relaxes the requirement and it only needs that the data contains no negative elements (Lee and Seung, 1999; Liu et al., 2006). Here we have used a modified form of NMF algorithm for decomposition of fMRI data into sources and corresponding time courses. Since the data has been made zero mean and white in order to decrease

Corresponding Author: Amir A. Khaliq, Department of Electronic Engineering, International Islamic University Islamabad, Pakistan

This work is licensed under a Creative Commons Attribution 4.0 International License (URL: http://creativecommons.org/licenses/by/4.0/) 
computational complexity, it does not obey the nonnegative constraint. Hence it can be called as Matrix Factorization (MF) algorithm.

In this study, we have proposed MF which is a modified version of NMF, with the advantage of fast convergence, quality of extracted sources and no permutations.

\section{NMF MODEL}

NMF model can be described as under. Suppose that $\boldsymbol{X}$ is the data matrix with non-negative elements. Then the NMF algorithm finds the decomposition of the data matrix into $A$ and $S$ such that:

$$
X \approx A . S
$$

With the constraint that the elements of both $A$ and $S$ are also non-negative (Lee and Seung, 1999).

For finding an approximation of the matrices $A$ and $S$, different cost functions are used in the literature, for example, Euclidean distance based and divergence or entropy based cost functions etc. The most common and simple is the Euclidean distance based as suggested by Lee and Seung (1999):

$$
J=\|X-A \cdot S\|^{2}
$$

This is now an optimization problem which can be solved with different techniques including gradient method, fixed point iteration methods etc. Authors of Lee and Seung (1999) have suggested multiplicative update equations for solving the NMF problem which is basically the extended version of gradient based update equations with a special multiplying factor. These update equations are depicted as under:

$$
\begin{aligned}
& \boldsymbol{S}(n+1)=\boldsymbol{S}(n) \otimes \frac{\left(\boldsymbol{A}^{T} \cdot \boldsymbol{X}\right)}{\left(\boldsymbol{A}^{T} \boldsymbol{A} . \boldsymbol{S}\right)} \\
& \boldsymbol{A}(n+1)=\boldsymbol{A}(n) \otimes \frac{\left(\text { X.S } \boldsymbol{S}^{T}\right)}{\left(\text { A.S.S } \boldsymbol{S}^{T}\right)}
\end{aligned}
$$

It should be noted that $\otimes$ and division in equation 3 and 4 are element by element.

\section{PROPOSED MF MODEL}

We are suggesting this proposed MF scheme for fMRI data which is not non-negative because of preprocessing step of centering and whitening. Centering of the observed data is done by subtracting mean from the observed data matrix $Z$ :

$$
\boldsymbol{X}^{c}=\boldsymbol{Z}-E[\boldsymbol{Z}]
$$

where,

$Z \quad=$ The observed data

$X^{C}=$ The centered data

Similarly whitening is done by multiplying the observed data with some whitening matrix $\mathrm{V}$ this process is done using the Eigen-decomposition (Friston, 1996) and is given as:

$$
\boldsymbol{X}=\boldsymbol{V} \boldsymbol{X}^{c}
$$

where, $\boldsymbol{V}=\boldsymbol{E} \boldsymbol{D}^{-1 / 2} \boldsymbol{E}^{T}$ and $\boldsymbol{E} \boldsymbol{D} \boldsymbol{E}^{T}=E\left[\boldsymbol{X}^{c} \boldsymbol{X}^{c T}\right], \mathrm{E}$ is the orthogonal matrix of eigenvectors of $\mathrm{E}\left[\mathrm{XX}^{\mathrm{T}}\right]$ and $\mathrm{D}$ is the diagonal matrix of its Eigen values.

The zero mean and whitening process transforms the observed data $Z$ into $X$ such that $\mathrm{E}\left[X X^{\mathrm{T}}\right]=\mathrm{I}$. Covariance of the source matrix $S$ will also become identity if and only if $\left[A A^{\mathrm{T}}\right]=\mathrm{I}$. This is the constraint which we need to implement in our proposed scheme. This also ensures that we are limiting our search only to the orthogonal matrices and thus a fast convergence is guaranteed.

Another preprocessing step which is specifically done in case of fMRI due to its high dimensional data is the dimension reduction step. Here we have used Singular Value Decomposition (SVD) as a dimension reduction technique (Hyvärinen et al., 2001). This data is now ready to be processed using the proposed scheme.

Starting from the optimization of NMF problem as mentioned in "NMF Model". Let the cost function be $J=\|\boldsymbol{X}-\boldsymbol{A} . \boldsymbol{S}\|^{2}$ which is to be minimized using the NMF update equations with the Ortho-normality constraint of the weight matrix $A$, such that $\left[A A^{\mathrm{T}}\right]=\mathrm{I}$.

To use this constraint the update equation of $S$ becomes:

$$
\boldsymbol{S}(n+1)=\boldsymbol{S}(n) \otimes \frac{\left(\boldsymbol{A}^{T} \cdot \boldsymbol{X}\right)}{(\boldsymbol{S})}
$$

It should be noted that the data now is not nonnegative, since for non-negative data, the condition of $\left[A A^{\mathrm{T}}\right]=\mathrm{I}$ cannot be fulfilled. Using equation 4 and 7 , fMRI data can be processed and hidden sources can be extracted with no non-negative data constraints.

To avoid the permutation problem which exists in NMF and most of the ICA algorithms, equation 4 needs to be re-derived for this tailored fMRI MF algorithm so that dependence of $A$ is minimized on $S$ during each iteration.

Starting from the cost function $J=\|\boldsymbol{X}-\boldsymbol{A} . \boldsymbol{S}\|^{2}$, this function will become zero when $X=A . S$, which can be written as $X X^{\mathrm{T}}=A . S X^{\mathrm{T}}$. Now writing the update equation for $A$ using the fixed point iteration. 

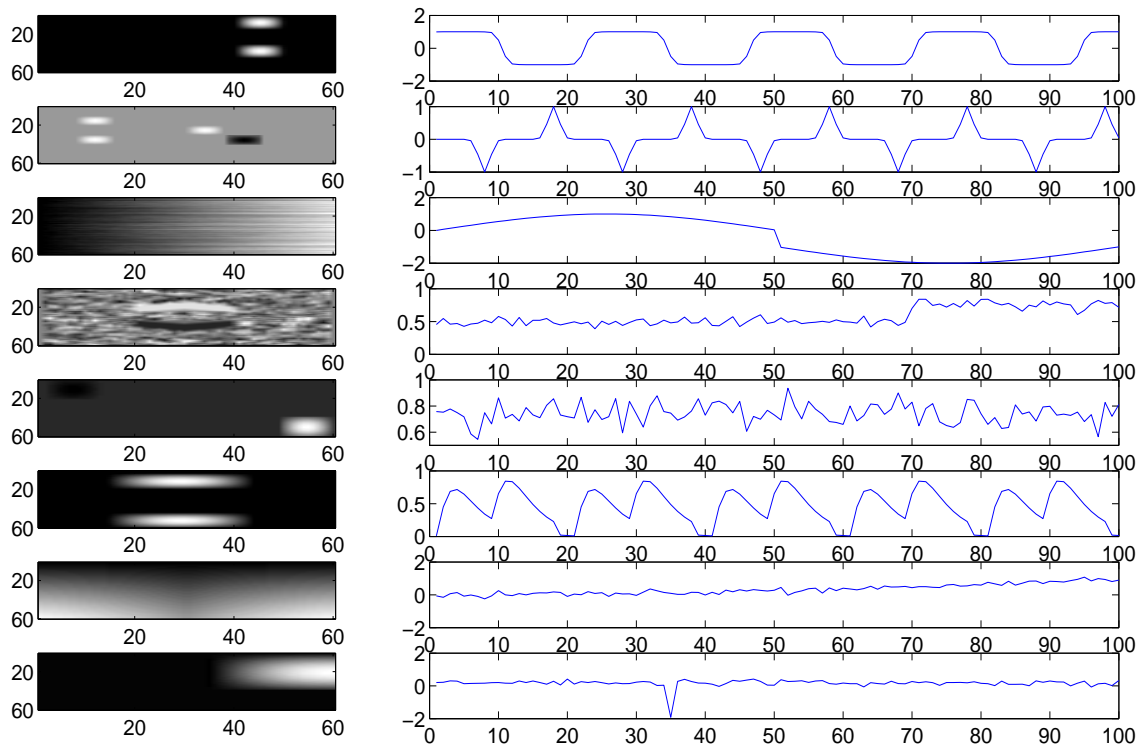

Fig. 1a: Simulated fMRI sources and time courses
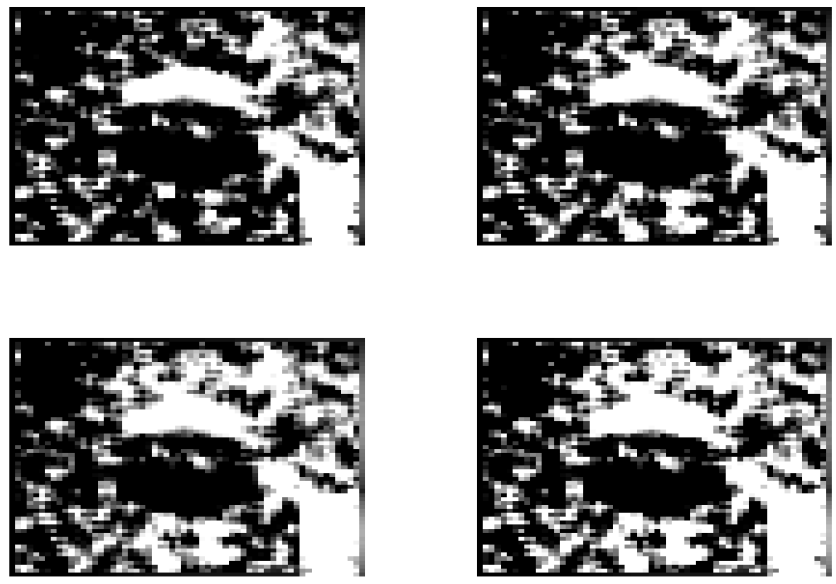

Fig. 1b: Four sample observed images

$$
\boldsymbol{A}(n+1)=\boldsymbol{A}(n)+\eta\left[\boldsymbol{X} \boldsymbol{X}^{T}-\boldsymbol{A} \boldsymbol{S} \boldsymbol{X}^{T}\right]
$$

where, $\eta$ is the learning rate. Now putting the learning rate $\eta=A / A S X^{\mathrm{T}}$ so that Eq. (8) becomes a multiplicative update equation as done in Lee and Seung (2011):

$$
A(n+1)=A(n) \otimes \frac{\left(X . X^{T}\right)}{\left(A . S . X^{T}\right)}
$$

This completes the derivation of the update equation for weight matrix $A$.

MF algorithm can be summarized as:

Step 1: Preprocess the fMRI data to get $X$ Step 2: Initialize Matrix $A$ and $S$ randomly

Step 3: Ortho-normalize $A$

Step 4: Update $A$ and $S$ using equation 7 and 9

Step 5: Stop if cost function $J$ is less than some small Step 6: End number; else go to Step 4

\section{SIMULATION RESULTS}

To validate and check the performance of the proposed MF algorithm, simulated fMRI like data is used which is freely available on web http://mlsp.umbc.edu/simulated-fMRI-data.html. This data was basically developed by Correa et al. (2005) for testing the validity of their algorithm. This data comprises of eight sources and their corresponding time courses as shown in Fig. 1a. The first source and corresponding time course is quite prominent in Fig. 1a which is representing functional activity. Source 2 and 
Res. J. Appl. Sci. Eng. Technol., 5(24): 5566-5571, 2013
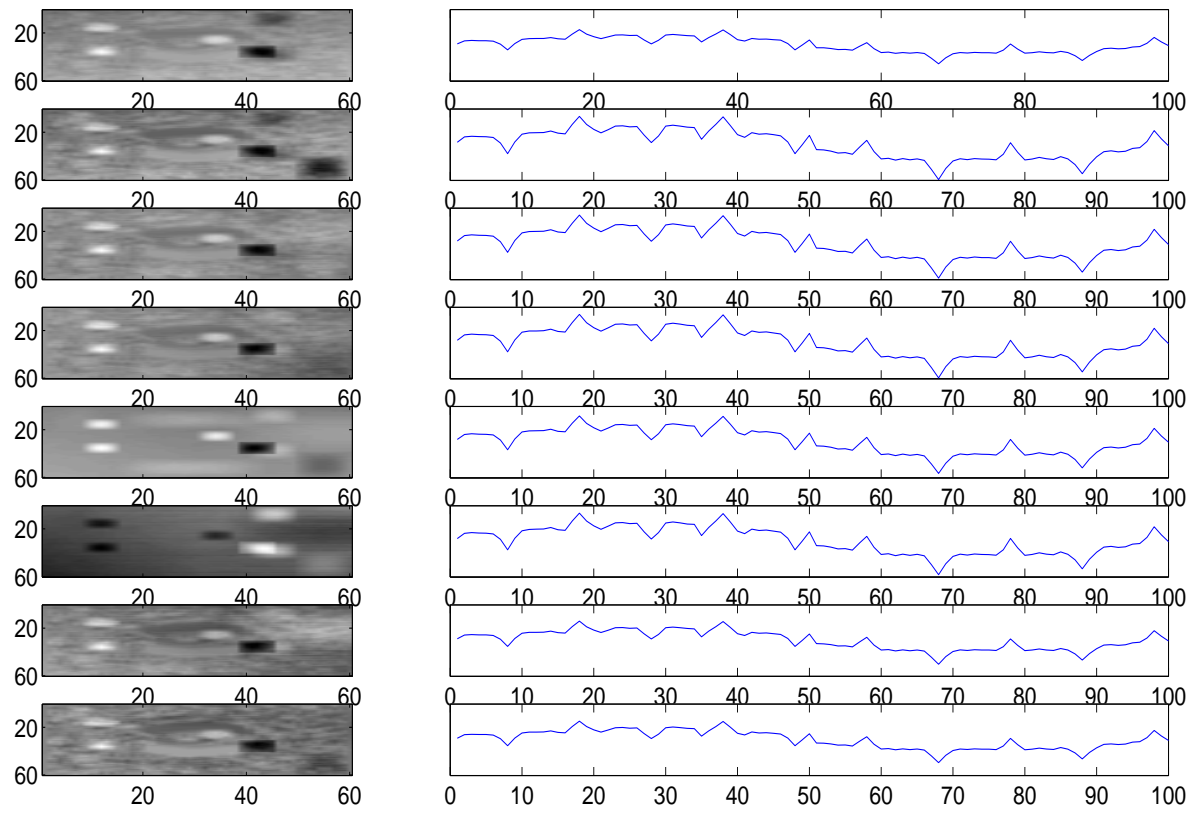

Fig. 2: Extracted sources using NMF
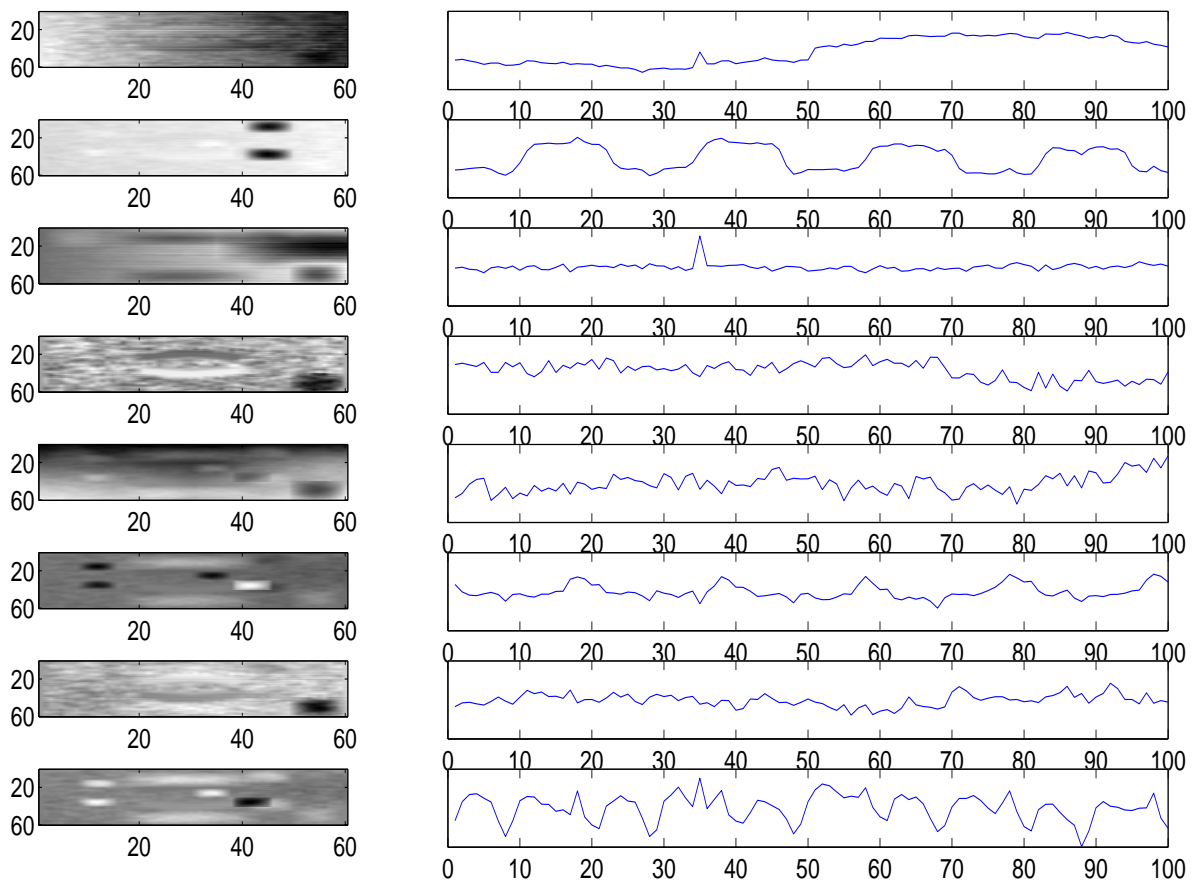

Fig. 3: Extracted sources using MF with permutations

source 6 represents the physiological tasks, while other sources and time courses show artifacts and noises. This data is mixed using linear combination of sources and their corresponding time courses. Four sample images of mixed data are shown in Fig. 1b. The mixed data is then preprocessed for further analysis.

Figure 2 shows extracted sources and time courses by NMF algorithm from the mixture data. It can be seen that sources and time courses are visually not recognizable and thus presenting a poor performance of NMF on fMRI data directly. Correlation results shown in Table 1 support this statement. Figure 3 shows extracted sources and time courses by using proposed matrix Factorization Algorithm (MF) that is equation 4 and equation 7 . It can be seen that extracted sources and time courses can be recognized by comparing it with 
Res. J. Appl. Sci. Eng. Technol., 5(24): 5566-5571, 2013
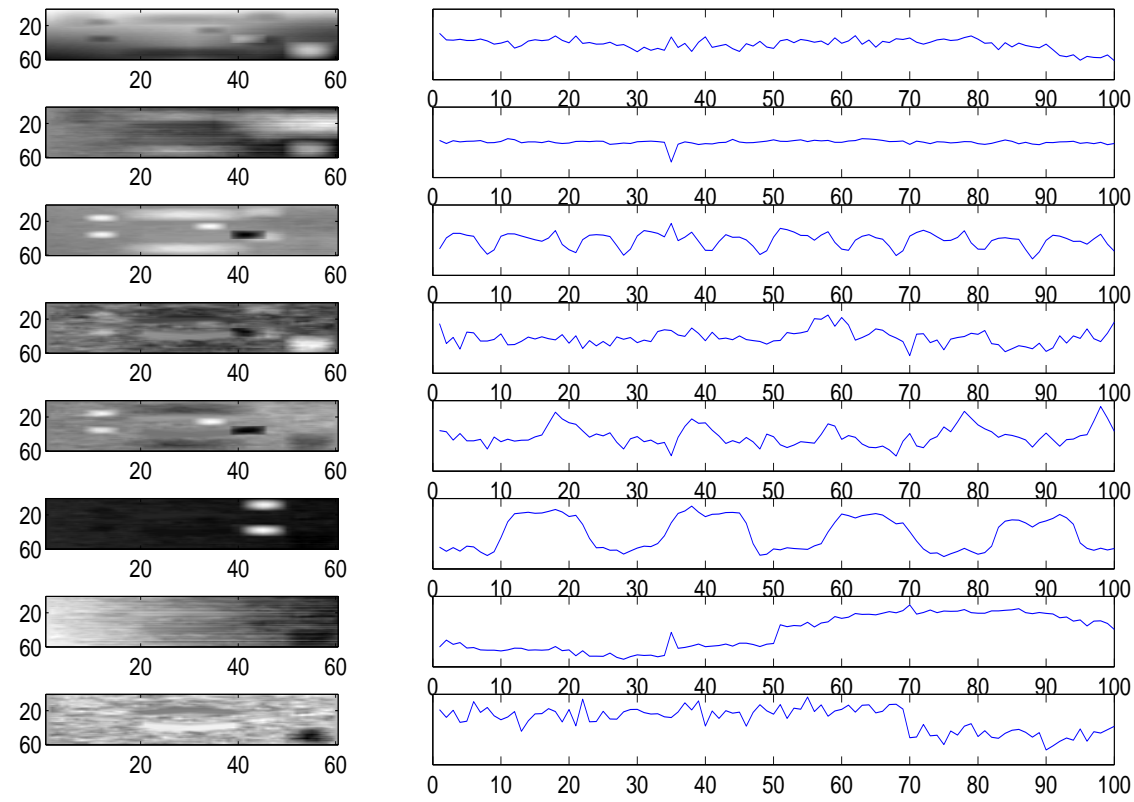

Fig. 4: Extracted sources using MF without permutations

Table 1: Execution time and performance of NMF, MF (WP) and MF (WO) in terms of correlation of extracted and actual sources and time courses

\begin{tabular}{llll}
\hline Scheme & NMF & MF (WP) & MF (WO) \\
\hline Execution time (sec) & 5 & 0.95 & 0.90 \\
S1/TC1 & $0.46 / 0.05$ & $0.96 / 0.97$ & $0.94 / 0.98$ \\
S2/TC2 & $0.68 / 0.43$ & $0.55 / 0.53$ & $0.69 / 0.76$ \\
S3/TC3 & $0.60 / 0.86$ & $0.96 / 0.98$ & $0.98 / 0.98$ \\
S4/TC4 & $0.45 / 0.44$ & $0.79 / 0.90$ & $0.77 / 0.94$ \\
S5/TC5 & $0.76 / 0.04$ & $0.65 / 0.43$ & $0.54 / 0.29$ \\
S6/TC6 & $0.29 / 0.01$ & $0.74 / 0.69$ & $0.71 / 0.72$ \\
S7/TC7 & $0.66 / 0.59$ & $0.92 / 0.53$ & $0.86 / 0.52$ \\
S8/TC8 & $0.31 / 0.30$ & $0.65 / 0.90$ & $0.64 / 0.91$ \\
Average correlation & $0.53 / 0.34$ & $0.78 / 0.74$ & $0.77 / 0.76$ \\
\hline
\end{tabular}

that of Fig. 1a. However permutation occurs by repeating the source extraction process.

Figure 4 shows sources and extracted time courses by using the proposed MF algorithm that is update equations 7 and 9. As discussed earlier permutation of vectors in the source matrix is stopped. Table 1 shows the performance of NMF, MF with permutation (MF $\mathrm{WP}$ ) and MF without permutation (MF WO) by correlating extracted (S/TC) sources/time courses with actual sources/time courses. It is evident that average correlation results of extracted sources/time courses with actual sources/time courses are $0.53 / 0.34$ with 5 sec convergence time. On the other hand proposed MFWP and MFWO gives average correlation results of $0.78 / 0.74$ and $0.77 / 0.76$ respectively.

\section{ACKNOWLEDGMENT}

We would like to acknowledge and thanks Machine learning and Signal Processing Lab University of Maryland Baltimore County USA for providing the simulated fMRI like data.

\section{CONCLUSION}

In this study NMF algorithm was tested directly on fMRI data. NMF update equation of source matrix was tailored as per Ortho-normality constraint which leads to relax the conditions of non-negativity on data matrix, source matrix and weight matrix. Simulation results show that it converges fast and shows quality in terms of average correlation between extracted and actual sources and time courses as compared to NMF. But this algorithm suffers from permutations as is NMF and ICA. Update equation of weight matrix is also modified so that its dependence on source matrix is minimized. This makes the process permutation free. Further study needs to be carried out to test the performance of MF on actual fMRI data and other similar problems.

\section{REFERENCES}

Backfrieder, W., 1996. Quantification of intensity variations in functional MR images using rotated principal component. Phys. Med. Biol., 4: 1425-1438.

Conceição, R.C., M. O'Halloran, M. Glavin and E. Jones, 2011. Evaluation of features and classifiers for classification of early stage breast cancer. $\mathrm{J}$. Electromagnet. Wave., 25(1): 1-14.

Correa, N., Y.O. Li, T. Adali and V. Calhoun, 2005. Comparison of blind source separation algorithms for fMRI using a new Matlab toolbox: GIFT. Proceeding of IEEE International Conference on Acoust., Speech, Signal Processing (ICASSP). Philadelphia, USA, 5: 401-403. 
Friman, O., J. Cadefamn, P. Lundberg, M. Borga and H. Knutsson, 2001. Detection of neural activity in functional MRI using canonical correlation analysis. Magn. Reson. Med., 45: 323-330.

Friston, K.J., 1996. Statistical Parametric Mapping and Other Analy-ses of Functional Imaging Data. In: Toga, A.W. and J.C. Mazziotta (Eds.), Brain Mapping: The Methods. Academic Press, San Diego, pp: 363-396.

Hyvärinen, A., J. Karhunen and E. Oja, 2001. Independent Component Analysis. Wiley, New York.

Khaliq, A.A., I.M. Qureshi and J.A. Shah, 2012. Temporal correlation-based spatial filtering of rician noise for functional MRIs. Chinese Phys. Lett., 29 018701, Doi: 10. 1088/0256-307x/29/1/ 018701.

Lee, D.D. and H.S. Seung, 1999. Learning the parts of objects by non-negative matrix factorization. Nature, 401: 788-791.

Lee, D.D. and H.S. Seung, 2011. Algorithms for nonnegative matrix factorization. Adv. Neural Info. Proc. Syst., 13: 556-562.

Liu, W., N. Zheng and O. You, 2006. Nonnegative matrix factorization and its applications in pattern recognition. Chinese Sci. Bull., 51(1): 7-18.

Mitra, P.P., S. Ogawa, X. Hu and K. Ugurbil, 1997. The nature of spatiotem-poral changes in cerebral hemo-dynamics as manifested in functional magnetic resonance imaging. Magn. Reson. Med., 37: 511-518.
Ogawa, S., T.M. Lee, A.R. Kay and D.W. Tank, 1990. Brain magnetic resonance imaging with contrast dependent on blood oxygenation. Proceedings of the National Academy of Sciences of the United States of America, 87: 9868-9872.

Torkkola, K., 1999. Blind separation for audio signals are we there yet? Proceedings of Workshop on Independent Component Analysis and Blind Signal Separation. France.

Vince, D.C., L. Jingyu and A. Tülay, 2009. A review of group ICA for fMRI data and ICA for joint inference of imaging, genetic and ERP data. Neuro Image, 45(1): 163-172.

Wei, D., L. Hualiang, L. Xi-Lin, V.D. Calhoun and T. Adali, 2011. ICA of fMRI data: Performance of three ICA algorithms and the importance of taking correlation information into account. Proceedings of the IEEE International Symposium on Biomedical Imaging: From Nano to Macro. Chicago, IL, pp: 1573-1576.

Xiaoxiang, W., T. Jie, L. Xingfeng, D. Jianping and A. Lin, 2004. Detecting brain activations by constrained non-negative matrix factorization from task-related BOLD fMRI. Proceedings of SPIE, 5369, Doi: 10.1117/12.5361.

$\mathrm{Yu}, \mathrm{C}$. and Z. Cheng, 2012. Research and simulation of FECG signal blind separation algorithm based on gradient method. Res. J. Appl. Sci. Eng. Technol., 4(16): 2707-2711. 